\title{
Manipulación del Plano Oclusal en Cirugía Ortognática: Consideraciones Faciales
}

\author{
Manipulation of the Oclusal Plane in Orthognathic Surgery: Facial Considerations
}

Sergio Olate* \& Henrique Duque de Miranda Chaves Netto**

OLATE, S. \& CHAVES NETTO, H. D. M. Manipulación del plano oclusal en cirugía ortognática: consideraciones faciales. Int. J. Odontostomat., 4(1):23-32, 2010.

RESUMEN: El plano oclusal ha sido un elemento importante en cirugía ortognática durante los últimos 20 años. Las investigaciones iniciales demostraron que las características faciales están altamente asociadas a la posición mandibular; siendo así, la rotación del plano oclusal permite una reposición oclusal diferente de la inicial, además una relación facial tanto vertical como anteroposterior favorable en términos de estética y proporciones faciales. Las investigaciones realizadas para cuantificar y caracterizar este movimiento han sido limitadas, pero auspiciosas; se ha determinado que la rotación del plano oclusal es un movimiento estable y versátil, siendo útil en diferente tipos de pacientes. El objetivo de este estudio es identificar las principales características asociadas a la rotación del plano oclusal así como analizar las principales investigaciones al respecto.

PALABRAS CLAVE: plano oclusal, manipulación del plano oclusal, rotación mandibular.

\section{INTRODUCCIÓN}

La cirugía ortognática ha ganado aceptación en el medio científico como un procedimiento estable y seguro (Proffit et al., 1996; Bailey et al., 2004). Las técnicas quirúrgicas utilizadas tanto en maxila como en mandíbula han sido consagradas como predecibles y versátiles siendo ejecutadas comúnmente por cirujanos maxilofaciales. El abordaje en maxila, claramente establecida por la osteotomía LeFort I, permite una adecuada movilización de los elementos óseos tanto en los movimientos de reposición anterior, inferior y superior. La osteotomía sagital de rama mandibular (OSRM), por otra parte, también permite movimientos tridimensionales, tanto en reposición antero-posterior e supero-inferior. Otras osteotomías mandibulares no son tan versátiles como la OSRM, requiriendo en algunos casos accesos extraorales y en otros casos injertos óseos para estabilizar el movimiento y optimizar la reparación de las osteotomías (Trauner \& Obwergeser, 1957; Bell et al., 1975; Bell \& Schendel, 1977; Drommer, 1986; Turvey \& Schardt-Sacco, 2000; Lupori et al., 2000).
En este sentido, la cirugía bimaxilar, empleando la osteotomía LeFort I y la OSRM, permite correcciones funcionales y estéticas con gran impacto en el complejo facial, determinando resultados en el sentido antero-posterior y en el sentido vertical que permite cubrir una parte importante de las demandas del paciente (Van Sickels \& D’Addario, 2007).

La situación anteroposterior (AP) en la evaluación facial del paciente es claramente influenciada por el perfil facial cóncavo o convexo, relacionados con los patrones oclusales clase II y clase III de Angle (Angle, 1899). A pesar que recientes estudio han demostrado limitadas diferencias proporcionales en morfometria mandibular en pacientes del biotipo clase II y clase III o entre diferentes géneros (Pozzer et al., 2009; Olate et al., 2009), cuando el patrón AP es analizado junto al patrón facial vertical, es posible obtener situaciones clínicas variadas y de complejo análisis. Estos padrones faciales fueron clasificados entonces como síndromes faciales cortos y largos.

\footnotetext{
Departamento de Odontologia Integral, Facultad de Medicina, Universidad de La Frontera, Chile.

** Práctica Privada, Juiz de Fora, Minas Gerais, Brasil.
} 
Los pacientes con síndrome facial corto históricamente fueron caracterizados por una dimensión vertical facial disminuida junto a un overbite aumentado en gran parte de los casos; el estudio de Opdebeeck \& Bell (1978) estableció las bases morfométricas de estos pacientes, demostrando con cefalometrias que efectivamente existía una dimensión vertical facial total (distancia entre puntos nasion y mentón, N-Me) disminuida junto a una dimensión vertical facial superior normal (distancia entre puntos nasion y espina nasal anterior, N-ENA). Los autores determinaron una dimensión vertical dentoalveolar reducida con aumento vertical de la rama mandibular y un ángulo mandibular (del punto gonion) reducido; sin embargo, el ángulo entre el plano craneal (silla turca-nasion,) y el plano mandibular (SN:PM) no siempre fue disminuido. Fue posible establecer relaciones donde la dimensión vertical facial disminuida seria asociada con aumento vertical de la rama mandibular.
Posteriormente, un nuevo artículo publicado por Opdebeeck et al. (1978) estableció las diferencias morfométricas y anatómicas mediante cefalometrias entre pacientes con síndrome facial corto y largo. La principal diferencia morfológica se asocio a la rotación mandibular, donde los pacientes con síndrome facial largo presentaban rotación mandibular hacia inferior y posterior (sentido horario) y en pacientes con síndrome facial corto con dirección anterior y superior (sentido antihorario) (Fig. 1); también fue observado cambios de la posición condilar, posiblemente asociados a la rotación mandibular. Esta rotación estaba acompañada por diferentes posiciones del hueso hioides y consecuentemente con diferentes posiciones de la lengua; la orofaringe también presentaba posiciones asociadas al hueso hioides y la mandíbula, con una significativa disminución en los pacientes con síndrome facial largo. Interesantemente, no existió diferencias en las mediciones del tercio medio facial expresada

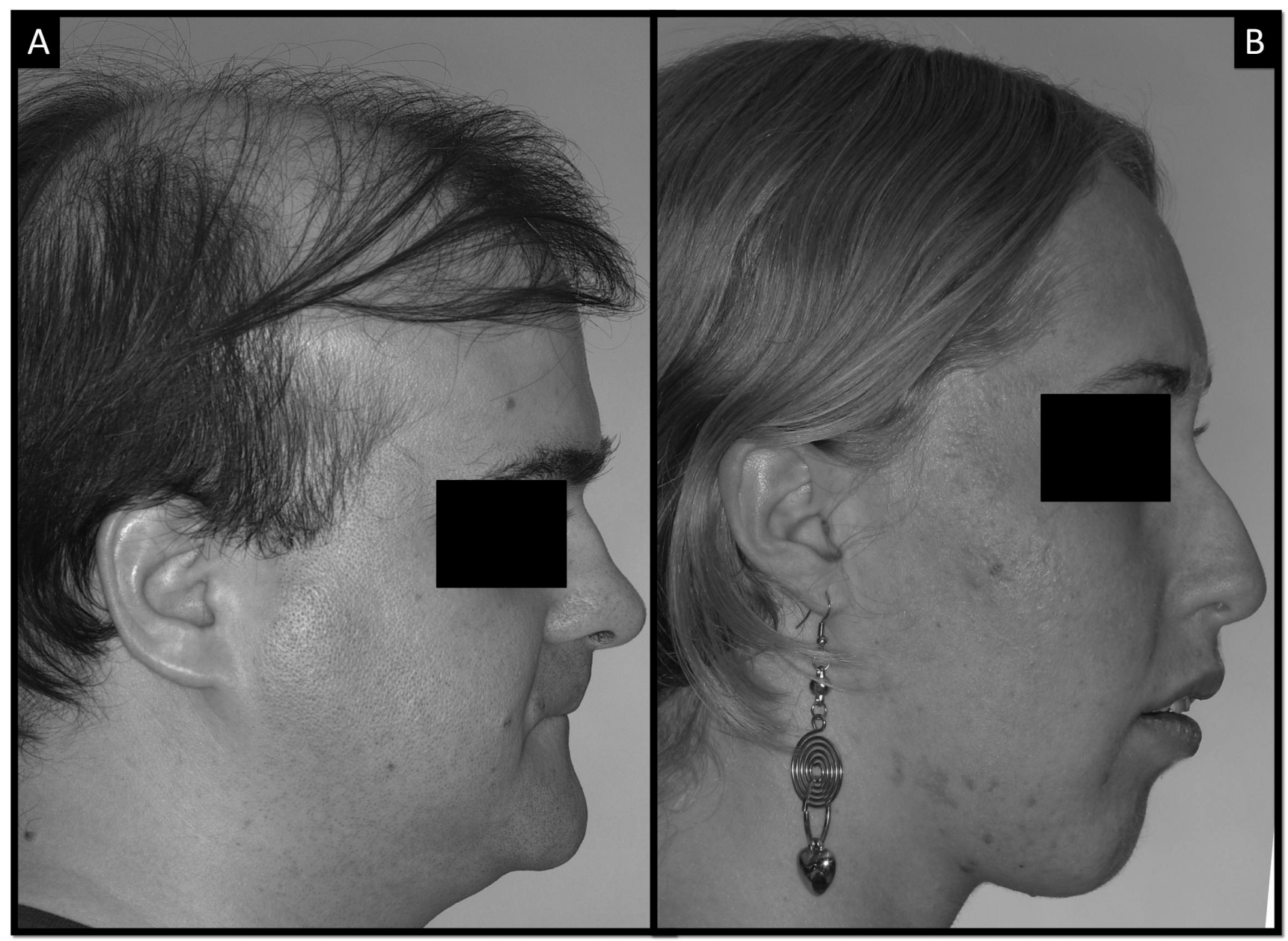

Fig. 1. A. Paciente con síndrome facial corto donde es posible identificar un perfil facial cóncavo y un plano mandibular cerrado; B. Paciente con síndrome facial largo identificando un perfil facial convexo y un plano mandibular abierto. 


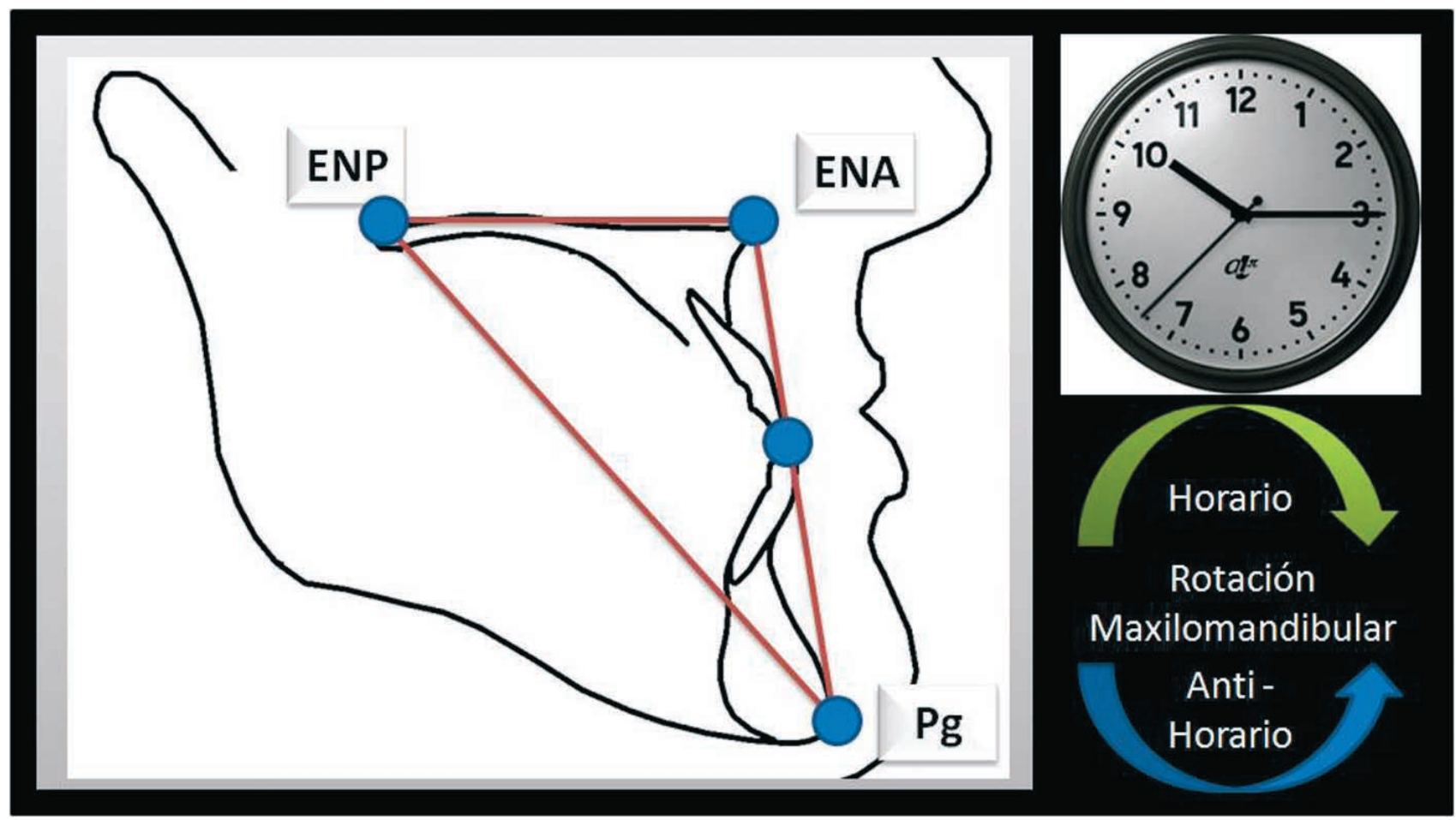

Fig. 2. Características de la rotación del plano oclusal en los sentidos horario y antihorario junto a los puntos anatómicos considerados en el movimiento: ENP=espina nasal posterior, ENA=espina nasal anterior, Pg=Pogonion.

por dimensiones asociadas a SN, espina nasal posterior (ENP), nasion (N) y espina nasal anterior (ENA).

Siendo así, fue posible concluir que la posición de la mandíbula gobernaría una parte importante de las deformidades faciales, permitiendo que Wolford et al. (1993) definiera la deficiencia anteroposterior de mandíbula como un síndrome, caracterizado también 3 subtipos donde el tipo I presentaría un bajo ángulo del plano mandibular, el tipo II un ángulo medio de plano mandibular y el tipo III un elevado ángulo de plano mandibular. En gran parte de estas incipientes investigaciones, el plano oclusal no era un elemento gravitante en las evaluaciones, no obstante era evidente que si se realizaba algún movimiento vertical o rotacional era altamente probable realizar modificaciones del plano oclusal, debido a la reposición mandibular.

El tratamiento de los pacientes con deformidades dentofaciales (DDF) asociadas a síndromes faciales cortos y largos fue entonces abordado con osteotomía LeFort I tanto para reposición superior o inferior de maxila; la mandíbula, por su parte, también presentaría algún movimiento rotacional, ya sea por autogiro o por osteotomía mandibular (Fig. 2) (Bell et al., 1977; Bell, 1977; Wolford \& Hilliard, 1981).
Es posible que el primer trabajo publicado sobre modificaciones del plano oclusal de forma intencional para cirugía ortognática sea el de McCollum et al. (1989), donde realizan una detallada descripción de los pacientes con características faciales del tipo clase II; los autores destacan que en la visión frontal existe un rostro "cuadrado" con un fuerte desarrollo de los músculos maseteros; en la visión lateral existe un mentón prominente y destacado, un labio inferior evertido por la alteración oclusal que frecuentemente es clase II división 1. Los hallazgos cefalométricos relatan paralelismos entre los planos mandibular, oclusal, palatal y base de cráneo y próximo del paralelismo también con el plano de Frankfort. McCollum et al. consideraron que el tratamiento debería incluir la mejoría de la maloclusión, aumento vertical del tercio facial inferior, estableciendo el balance entre labios y una adecuada posición del mentón. Con estos elementos, los autores diseñaron una evaluación geométrica donde se realizaría un cambio en la angulación del plano oclusal, aumentando el ángulo del mismo mediante la reposición superior de una región posterior de la maxila con un eje de fulcro ubicado en la región maxilar anterior. Con esta rotación mandibular (en el sentido horario), los beneficios estéticos son relacionados a un aumento del ángulo nasolabial, aumento de la proyección anteroposterior de la región paranasal, reducción 
de la eversión del labio inferior, reducción de la proyección anteroposterior del mentón y aumento de la dimensión vertical facial del tercio inferior.

Después de estos estudios, Wolford et al. (1993) establecieron patrones faciales en relación al plano oclusal, analizando los objetivos quirúrgicos tanto faciales como oclusales y las posibles modificaciones del plano oclusal en cirugía ortognática (Tabla I).

Plano Oclusal (PO). Gran parte de los estudios cefalométricos trazan el plano oclusal como una línea que intercepta el punto de contacto de molares y el punto medio del overbite o la mordida abierta del paciente (Ricketts, 1981). El reconocimiento del PO parece sencillo, pero cuando el clínico se enfrenta a un paciente con alteraciones en la curva oclusal, mordida abierta o maloclusión severa el reconocimiento del PO escapa a la norma; en estos casos, Reyneke (1998) sugiere trabajar con dos $\mathrm{PO}$ que serian el maxilar y el mandibular, donde ambos serian tangentes a la superficie oclusal del primer molar y superficie incisal de incisivo central siendo completamente aplicables en la planificación quirúrgica. Los valores normales del PO para pacientes clase I sin DDF según Ricketts está en una relación de $8^{\circ} \pm 5^{\circ}$ con el plano de Frankfort y según Steiner (1959), $14^{\circ}$ cuando se relaciona con la base de cráneo.

Los primeros trabajos de Wolford et al. (1993, 1994) en relación al manejo del plano oclusal señalan la importancia de reconocerlo correctamente. La situación clínica muchas veces muestra un paciente con una posición maxilar adecuada y significativa deficiencia mandibular y a la hora de realizar el análisis cefalométrico, las observaciones de profundidad maxilar y profundidad mandibular invierten los análisis clínicos. Esta situación, según Wolford et al. (1993), hace necesario corregir la posición del plano de Frankfort, ya que la posición diseñada sobre la radiografía lateral puede estar alterada por la incorrecta elección de los puntos infraorbitario, porion y nasion; es decir, el análisis cefalométrico se debería adaptar al análisis facial. El artículo de discusión de Epker (1977) limita la indicación propuesta por Wolford et al. (1993), señalando que si existiese una corrección del plano de Frankfort podría perfectamente corregirse el trazado cefalométrico en su totalidad a conveniencia del cirujano, o bien podría parcializarse del análisis pre quirúrgico del paciente.

En el mismo trabajo, Wolford et al. $(1993,1994)$ señalan que los tres elementos más importantes en el análisis cefalométrico lateral son la 1) posición vertical del incisivo central, 2) la posición AP de maxila e incisivo y 3) angulación del plano oclusal; sin duda, estos tres elementos son gravitantes para determinar la rotación del plano oclusal, que finalmente sería guiado por las necesidades faciales y oclusales del paciente.

Manipulación del PO y del Complejo Maxilomandibular (CMM). La manipulación del PO

Tabla I. Características faciales y oclusales en pacientes que presentan un plano oclusal (PO) aumentado o disminuido según Wolford et al. (1993).

\begin{tabular}{|c|c|c|}
\hline & Tipo facial con PO disminuido & Tipo facial con PO elevado \\
\hline Plano oclusal & Bajo (menos de $4^{\circ}$ ) & Elevado (mayor que $12^{\circ}$ ) \\
\hline Plano mandibular & Bajo & Elevado \\
\hline Angulo mandibular & Bien definido & - \\
\hline Mentón & Bien definido y proyectado & Poca proyección \\
\hline Altura vertical & Disminuida & $\begin{array}{l}\text { Aumentada (algunos casos con } \\
\text { deficiencia posterior) }\end{array}$ \\
\hline Angulación incisivo maxilar & $\begin{array}{l}\text { Disminuido (algunos casos con } \\
\text { sobre angulación) }\end{array}$ & $\begin{array}{l}\text { Disminuido (algunos casos con } \\
\text { sobre angulación) }\end{array}$ \\
\hline Angulación incisivo mandibular & Disminuida & Aumentada \\
\hline Clasificación de Angle & $\begin{array}{l}\text { Clase II (algunos casos clase I o } \\
\text { III) }\end{array}$ & $\begin{array}{l}\text { Clase II (algunos casos clase I o } \\
\text { III) }\end{array}$ \\
\hline Curva de oclusión (spee) & $\begin{array}{l}\text { Acentuada en mandíbula y } \\
\text { algunos casos invertido en }\end{array}$ & Acentuada \\
\hline Overbite & Profundo & Mordida abierta anterior \\
\hline
\end{tabular}


exige una cirugía bimaxilar y la elección de un punto de rotación reproducible en los trazados cefalométricos y el la cirugía de modelos; el eje de rotación y el movimiento del CMM se encuentra dentro del triangulo producido por los puntos de la ENP, ENA y el pogonion (Pg) (Fig. 2), donde la elección del punto de rotación dependerá de los resultados deseados. Reyneke establece algunos parámetros útiles para estudiar la rotación tanto en sentido horario como en el sentido antihorario (Tabla II), mientras que Wolford et al. (1993) señalan que los objetivos quirúrgicos para los pacientes con plano oclusal disminuido son:

1. Aumento del plano oclusal

2. Aumento del plano mandibular

3. Disminución ángulo incisivo maxilar

4. Rotación posterior del mentón en relación con incisal de incisivo mandibular

5. Disminución posterior de la altura facial

6. Avance de las estructuras óseas paranasales

Mientras que en pacientes con plano oclusal elevado, los objetivos quirúrgicos son:

1. Disminución del plano oclusal

2. Disminución del plano mandibular

3. Aumento de la angulación de incisivo maxilar

4. Disminución de la angulación incisivo mandibular

5. Aumento de la proyección AP del mentón en relación con incisivo mandibular

6. Aumento del ángulo mandibular

7. Establecimiento de guía incisal

8. Aumento de la altura facial posterior

9. Retroposicón del área paranasal en relación con el incisivo maxilar
La cirugía bimaxilar permite atingir estos elementos (Fig. 3). Como Van Sickels \& D'Addario señalan, en casos de DDF que presenten más de un plano oclusal (plano oclusal maxilar y plano oclusal mandibular) se debe buscar un tercer plano de forma quirúrgica. La manipulación del PO, entonces, mejoraría la proyección del mentón en el sentido deseado y evitaría cirugías que signifiquen reposición de mentón o genioplastias.

El movimiento estimado con el análisis facial debe ser evaluado también con los trazados de previsión cefalométrica. Cuál será la mejor opción en el manejo del PO debe ser estudiada con diferentes trazados de previsión; en este sentido, cuando se escoge un punto de rotación como por ejemplo la ENP, se debe considerar el movimiento que presentaran molares e incisivos, debido a que son ellos los considerados en la cirugía de modelos. De este modo, si se planifica, por ejemplo, una reposición superior de 5 $\mathrm{mm}$ posterior, manteniendo la altura vertical en los incisivos, esos $5 \mathrm{~mm}$ deben ser estimados en el punto oclusal del primer molar superior, ya que una reposición superior de $5 \mathrm{~mm}$ en la ENP llevara a una reposición menor que $5 \mathrm{~mm}$ en el punto oclusal del primer molar superior.

La ortodoncia pre-quirúrgica es extremamente importante en estos casos, ya que generalmente dicta las posibilidades del movimientoquirúrgico y de la estabilidad del mismo (Jacobs \& Sinclair, 1983). Pacientes con bajo plano oclusal deberían presentar aumento en la angulación de incisivos maxilar y disminución de la angulación de incisivos mandibulares, mientras que pacientes con elevado plano oclusal deberían pre-

Tabla II. Movimiento estimados en los diferentes elementos del complejo maxilomandibular cuando se realiza una rotación en el sentido horario o antihorario según Reyneke (1998).

\begin{tabular}{|c|c|c|c|c|}
\hline & $\begin{array}{l}\text { Rotación horaria } \\
\text { en relación a l a } \\
\text { ENA }\end{array}$ & $\begin{array}{l}\text { Rotación horaria } \\
\text { posterior a la } \\
\text { ENA }\end{array}$ & $\begin{array}{l}\text { Rotación } \\
\text { antihoraria en } \\
\text { relación a la } \\
\text { ENA }\end{array}$ & $\begin{array}{l}\text { Rotación } \\
\text { antihoraria } \\
\text { posterior a la } \\
\text { ENA }\end{array}$ \\
\hline Angulo plano oclusal & Aumenta & Aumenta & Disminuye & Disminuye \\
\hline Angulo plano mandibular & Aumenta & Aumenta & Disminuye & Disminuye \\
\hline Angulo & Aumenta & Aumenta & Disminuye & Disminuye \\
\hline Angulo incisivo maxilar & Disminuye & Disminuye & Aumenta & Aumenta \\
\hline Proyección AP Pogonion & Disminuye & Disminuye & Aumenta & Aumenta \\
\hline Exposición & Aumento leve & Aumenta & Aumento leve & Disminuye \\
\hline Altura facial inferior & Aumenta & Aumenta & Disminuye & Disminuye \\
\hline
\end{tabular}




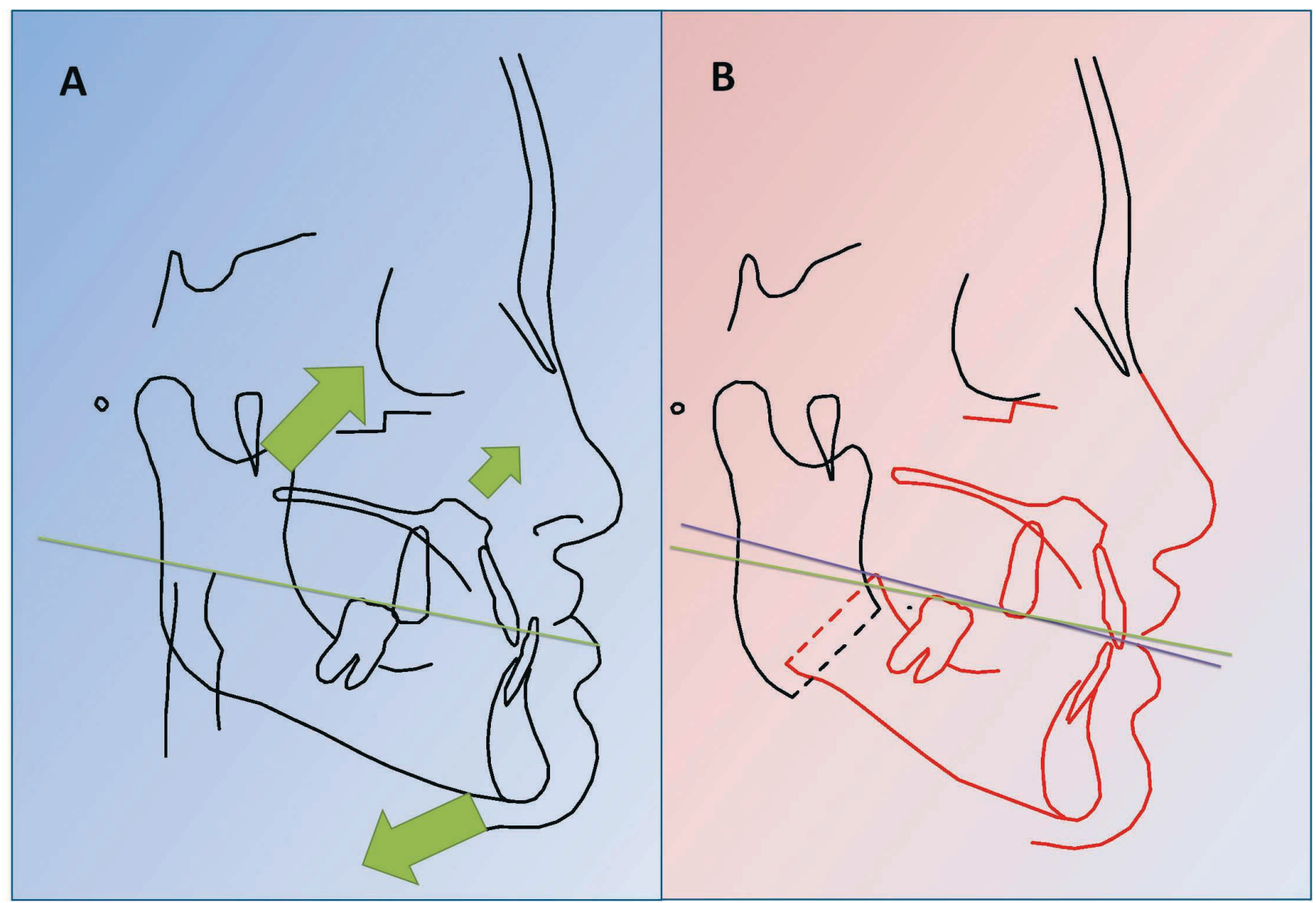

Fig. 3. Diseño radiográfico de una paciente con características faciales clase III. A. Diagnostico representado por un exceso antero-posterior de mandíbula e deficiencia antero-posterior de maxila. B. El trazado de previsión demuestra el cambio en la angulación del plano oclusal con sentido horario permitiendo una armónica posición de Pg y aumento de la dimensión facial vertical inferior.

sentar incisivos maxilares con disminuida angulación e incisivos mandibulares con aumentada angulación. La planificación de rotación horaria o antihoraria debe ser, por lo tanto, identificada en conjunto con la planificación ortodóncica prequirúrgica. En segmentaciones maxilares, la angulación de incisivos no es tan crítica (Wolford et al., 1994).

Una de las grandes dudas en estos movimientos esta en relación al efecto sobre la ATM; de nuestro conocimiento, el único estudio disponible a la fecha es el de Cottrell et al. (1997), quienes desarrollaron una investigación en 22 pacientes con ATM clínicamente saludable con una disminución quirúrgica mínima de $5^{\circ}$ del PO (media de $8,9^{\circ}$ ) en relación al plano de Frankfort; el promedio de avance en Pg fue de $12 \mathrm{~mm}$ $(6 \mathrm{~mm}$ a $21 \mathrm{~mm})$; fueron realizadas radiografías y tomografías postquirúrgicas con un seguimiento medio de 30,6 meses. Los autores relatan disminución del espacio articular superior y posterior $(14 \%$ y $18 \%)$ sin modificaciones en la región articular anterior; estas modificaciones fueron observadas de forma precoz dentro de los primeros 21 días. Después de este tiempo, las modificaciones articulares fueron menores. La remodelación condilar fue mínima y relativa al tamaño del cóndilo. Los autores concluyen que el grado de cambio del PO no tiene relación con la cantidad de remodelación condilar o con la posición condilar dentro del espacio articular y que la rotación del PO puede ser uno de múltiples factores asociados a la reabsorción condilar.

Estabilidad de los Resultados. Proffit et al. señalan que la estabilidad posquirúrgica se relaciona con 1) la presencia de tejido muscular libre de tensión (intraoperatoria), 2) adaptación muscular (postoperatorio) y 3) con el hecho de que la adaptación muscular es realizada principalmente con la longitud del musculo y no con el cambio en la orientación del mismo. Por su parte, Reyneke señalaba que el principal motivo de recidiva 
en rotación antihoraria estaba asociada al cirujano cuando este intentaba realizar la rotación conjunta del segmento proximal de la osteotomía mandibular; cuando no es realizado este movimiento, no debería existir grandes recidivas quirúrgicas.

Chemello et al. (1994) realizaron un estudio en 41 pacientes con cirugías bimaxilares en ausencia de signos o síntomas de alteraciones de ATM con un acompañamiento mínimo de 12 meses y con osteosíntesis a través de fijación interna rígida (FIR); 14 pacientes fueron sometidos a aumento del PO y 27 pacientes fueron sometidos a disminución del mismo. En pacientes con aumento quirúrgico del $\mathrm{PO}$ fue realizada una rotación aproximada de 5,6o con una reposición anterior de la maxila de aproximadamente $3,3 \mathrm{~mm}$ en el punto $\mathrm{A}$; el primer molar superior fue impactado en 2,5mm aproximadamente; prácticamente no existió movimiento horizontal del punto $\mathrm{B}$ mandibular mientras que el $\mathrm{Pg}$ fue ubicado en una retroposición de $2 \mathrm{~mm}$. En el grupo de disminución quirúrgica del PO hubo una media de $-8,8^{\circ}$ de inclinación del plano oclusal; el primer molar superior prácticamente no tuvo movimiento vertical mientras que en la región anterior existió una reposición superior de $3 \mathrm{~mm}$ aproximadamente; el punto A tuvo un avance de $1,3 \mathrm{~mm}$, mientras que en el punto $B$ mandibular presento un avance de $8 \mathrm{~mm}$ y $10 \mathrm{~mm}$ en el $\mathrm{Pg}$. Interesantemente, la altura vertical de la rama mandibular no sufrió alteraciones en las evaluaciones prequirúrgicas y postquirúrgicas. Los autores señalan ausencia de recidiva postquirúrgica debido principalmente a la desinserción de los músculos pterigoideo medial y masetero durante la cirugía, lo que permitiría a readaptación muscular y reposición ósea sin stress.

En un estudio retrospectivo de 88 pacientes Reyneke et al. (2007) estudiaron el resultado de cirugías bimaxilares. La maxila fue abordada con osteotomía LeFort I y FIR con sistema 1.5, mientras que la mandíbula fue abordada con OSRM bilateral fijadas con 3 tornillos bicorticales del sistema 2.0. El grupo 1 (25 pacientes) fue tratado por auto rotación de mandíbula, el grupo 2 (25 pacientes) fue tratado con rotación horaria del CMM y el grupo 3 (41 pacientes) fue tratado por rotación antihoraria del CMM. La estabilidad posquirúrgica analizada en el largo plazo no presento diferencias entre los grupos tanto en las evaluaciones anteroposteriores como en las evaluaciones verticales; se evidencio una tendencia a recidiva en el sentido contrario del movimiento, mas no existió relación estadística entre los valores (Tabla III).

Gonçalves et al. (2008) publicaron un estudio retrospectivo en 72 pacientes con una angulación mínima del PO de $14^{\circ}$ y con características faciales de deficiencia anteroposterior de maxila y mandíbula y dimensión vertical aumentada. Se dividieron tres grupos con cirugía ortognática bimaxilar, siendo el primero determinado por ATM saludable (21 pacientes), el segundo con evidencia de desplazamiento de disco mediante resonancia magnética realizando cirugía ortognática conjunta con reposición de disco articular (35 pacientes) y el tercer grupo con evidencia clínica y en resonancia magnética de desplazamiento de disco sin cirugía articular conjunta (16 pacientes). Gonçalves et al. (2008) observaron que en el grupo 1 y grupo 2 existió estabilidad posquirúrgica del movimiento, mientras que en el grupo 3 existió un $28 \%$ de recidiva en la evaluación antero-posterior; además, el $84 \%$ presento dolor articular después del segundo año de seguimiento y el $48 \%$ debió ser sometido a una nueva cirugía ortognática. Los autores sugieren que la rotación antihoraria del CMM aumenta las cargas en la ATM debido a la fuerza que ejercen los músculos, periostio y piel y que pacientes con trastornos témporomandibulares presentan tendencias a recidiva del tratamiento quirúrgico.

Tabla III. Resultados obtenidos en el estudio de Reyneke et al. (2007) en 88 pacientes sometidos a tres tipos de movimientos (convencional, rotación horaria y rotación antihoraria) $(\mathrm{Mx}=$ maxila, $\mathrm{Md}=$ mandíbula, $\mathrm{PO}=$ plano oclusal, $\mathrm{CCM}=$ complejo maxilomandibular).

\begin{tabular}{|c|c|c|c|}
\hline & $\begin{array}{l}\text { Tratamiento } \\
\text { convencional }\end{array}$ & $\begin{array}{l}\text { Rotación horaria del } \\
\text { CMM }\end{array}$ & $\begin{array}{l}\text { Rotación antihoraria } \\
\text { del CMM }\end{array}$ \\
\hline Avance $\mathrm{Mx}$ & $2,24 \mathrm{~mm}$ & $2,73 \mathrm{~mm}$ & $3,32 \mathrm{~mm}$ \\
\hline Reposición superior Mx & $1,91 \mathrm{~mm}$ & $1,17 \mathrm{~mm}$ & $-3,84 \mathrm{~mm}$ \\
\hline Reposición posterosuperior de & $0 ; 32 \mathrm{~mm}$ & $1,68 \mathrm{~mm}$ & $1,07 \mathrm{~mm}$ \\
\hline Avance Md & $7 ; 26 \mathrm{~mm}$ & $0,24 \mathrm{~mm}$ & $10,81 \mathrm{~mm}$ \\
\hline Angulo PO & $-0,32^{\circ}$ & $4,89^{\circ}$ & $-4,97^{\circ}$ \\
\hline Angulo Plano Md & $-1,62^{\circ}$ & $5,22^{\circ}$ & $-4,06^{\circ}$ \\
\hline
\end{tabular}


La rotación del CMM hacia anterior y superior lleva a la reposición del tubérculo geniano y consecuentemente a una reposición anterior del hueso hioides y aumento del espacio aéreo en la faringe. Mehra et al. (2001) realizaron un estudio en 50 pacientes con elevado plano oclusal (mayor que 12o) para reposición anterior bimaxilar, con FIR y sin bloqueo inter maxilar; los autores presentan un significativo aumento de la vía aérea faríngea y en la posición anterior de la lengua, donde la rotación antihoraria aumento $76 \%$ la vía aérea en pacientes con avance de mandíbula y en $65 \%$ en paciente que recibieron una reposición posterior de mandíbula. El paladar blando tuvo un avance aproximado del $47 \%$ en los casos de reposición anterior o posterior de mandíbula.

Gonçalves et al. (2006) también reportaron satisfactorios resultados en 56 pacientes cuando realizaron la rotación antihoraria del PO para aumentar la vía aérea; los autores relatan aumento en las dimensiones de las regiones inferiores y medias de la orofaringe en el corto y largo plazo, estableciendo un avance de $\mathrm{Pg}$ de $3,1 \mathrm{~mm}$ mas anterior que el punto $\mathrm{B}$ y $6,2 \mathrm{~mm}$ mas anterior que el punto incisal del incisivo mandibular.

Como conclusión, existe limitada información respecto de los resultados a largo plazo de la rotación del CMM. Gran parte de los estudios son retrospectivos y desarrollados por un mismo grupo de autores, aumentando el sesgo de los estudios. De hecho, hace prácticamente 20 años que Wolford y Reyneke exploraron este concepto y actualmente son esos autores quienes han estudiado el efecto de la manipulación del PO en la estética y la función. Sin dudas rotaciones del CMM traen beneficios estéticos en el paciente con DDF, disminuyendo muchas veces la cantidad de cirugía (puede eliminar la genioplastia), pero aun existen dudas como por ejemplo ¿hasta cuanto es posible cambiar la angulación del plano oclusal? o ¿cual es el real efecto de la rotación del CMM con la dimensión vertical posterior? o cuáles son las consecuencias articulares a largo plazo en pacientes con rotación del CMM?.

OLATE, S. \& CHAVES NETTO, H. D. M. Manipulation of the oclusal plane in orthognathic surgery: facial considerations. Int. J. Odontostomat., 4(1):23-32, 2010.

ABSTRACT: Occlusal plane has been an important element in orthognatic surgery during these last 20 years. The primary investigations have shown that facial characteristics are highly associated to mandibular positioning. Thus, occlusal plane rotation allows an acclusal plan different from the initial one, even as vertical and anterior-posterior positioning more favorable en endings of aesthetic. Besides the studies carried through to measure y to characterize these movements are limited, they are favorable. They have demonstrated that occlusal plane rotation versatile y stable, being useful en different types of facial deformities. The meaning of this work are identify the more important characteristic about occlusal plane rotation as well analyze the most cited articles in the literature.

KEY WORDS: occlusal plane, occlusal plane manipulation, mandibular rotation.

\section{REFERENCIAS BIBLIOGRÁFICAS}

Angle, E. H. Classification of malocclusion. Dent. Cosmos, 41:248-64, 1899.

Bailey, L. J.; Cevidanes, L. H. \& Proffit, W. R. Stability and predictability of orthognathic surgery. Am. J. Orthod. Dentofacial Orthop., 126(3):273-7, 2004.

Bell, W. H. Correction of the short-face syndrome vertical maxillary deficiency: a preliminary report. J. Oral Surg., 35(2):110-20, 1977.

Bell, W. H.; Creekmore, T. D. \& Alexander, R. G. Surgical correction of the long face syndrome. Am J. Orthod., 71(1):40-67, 1977.
Bell, W. H.; Fonseca, R. J.; Kennedy, J. W. \& Levy, B. $M$. Bone healing and revascularization after total maxillary osteotomy. J. Oral Surg., 33(4):253-60, 1975.

Bell, W. H. \& Schendel, S. A. Biologic basis for modification of the sagittal ramus split operation. $J$. Oral Surg., 35(5):362-9, 1977.

Chemello, P. D.; Wolford, L. M. \& Buschang, P. H. Occlusal plane alteration in orthognathic surgery-Part II: Long-term stability of results. Am. J. Orthod. Dentofacial Orthop., 106(4):434-40, 1994. 
Cottrell, D. A.; Suguimoto, R. M.; Wolford, L. M.; Sachdeva, R. \& Guo, I. Y. Condylar change after upward and forward rotation of the maxillomandibular complex. Am. J. Orthod. Dentofacial Orthop., 11(2):156-62, 1997.

Drommer, R. B. The history of the "Le Fort I osteotomy". J. Maxillofac. Surg., 14(3):119-22, 1986.

Epker, B. N. Modification in the sagittal osteotomy of the mandible. J. Oral Surg., 35:157-9, 1977.

Gonçalves, J. R.; Buschang, P. H.; Goncalves, D. G. \& Wolford, L. M. Postsurgical stability of oropharyngeal airway changes following counterclockwise maxillo-mandibular advancement surgery. J. Oral Maxillofac. Surg., 64(5):755-62, 2006.

Gonçalves, J. R.; Cassano, D. S.; Wolford, L. M.; Santos-Pinto, A. \& Márquez, I. M. Postsurgical stability of counterclockwise maxillomandibular advancement surgery: affect of articular disc repositioning. J. Oral Maxillofac. Surg., 66(4):72438, 2008.

Jacobs, J. D. \& Sinclair, P. M. Principles of orthodontic mechanics in orthognathic surgery cases. Am. J. Orthod., 84(5):399-407, 1983.

Lupori, J. P.; Kewitt, G. \& Van Sickels, J. E. Bilateral sagittal split osteotomy: advancement and setback. In Fonseca, R.; Betts, N. \& Turvey, T. Oral and Maxillofacial Surgery. Vol 2. Phyladelphia: WB Saunders Company, 2000. pp.297-310.

McCollum, A. G.; Reyneke, J. P. \& Wolford, L. M. An alternative for the correction of the Class II low mandibular plane angle. Oral Surg. Oral Med. Oral Pathol., 67(3):231-41, 1989.

Mehra, P.; Downie, M.; Pita, M. C. \& Wolford, L. M. Pharyngeal airway space changes after counterclockwise rotation of the maxillomandibular complex. Am. J. Orthod. Dentofacial Orthop., 120(2):154-9, 2001.

Olate, S.; Pozzer, L.; Sawazaki, R.; Asprino, L. \& de Moraes M. ¿Existen diferencias em la morfometria mandibular de pacientes candidatos a cirugia ortognatica?. Parte 2: Influencias dal género. Int. J. Morphol., 27(3):777-781, 2009.
Opdebeeck, H. \& Bell, W. H. The short face syndrome. Am. J. Orthod., 73:499-511, 1978.

Opdebeeck, H.; Bell, W. H.; Eisenfeld, J. \& Mishelevich, D. Comparative study between the SFS and LFS rotation as a possible morphogenic mechanism. Am. J. Orthod., 74(5):509-21, 1978.

Pozzer, L.; Olate, S.; Asprino, L. \& de Moraes M. $¿$ Existen diferencias em la morfometria mandibular de pacientes candidatos a cirugia ortognatica?. Parte 1: influencias da la clase facial. Int. J. Morphol., 27(3):751-6, 2009.

Proffit, W. R.; Turvey, T. A. \& Phillips, C. Orthognatic surgery: a hierarchy of stability. Int. J. Adult Orthodon. Orthognath. Surg., 11(3):191-204, 1996.

Reyneke, J. P. Surgical manipulation of the oclusal plane: new concepts in geometry. Int. J. Adult Orthodon. Orthognath. Surg., 13(4):307-16, 1998.

Reyneke, J. P.; Bryant, R. S.; Suuronen, R. \& Becker, P. J. Postoperative skeletal stability following clockwise and counter-clockwise rotation of the maxillomandibular complex compared to conventional orthognathic treatment. Br. J. Oral Maxillofac. Surg., 45(1):56-64, 2007.

Ricektts, R. M. Perspectives in the clinical application of cephalometrics. The first fifty years. Angle Orthod., 51:115-50, 1981.

Steiner, C. C. Cephalometrics in clinical practice. Angle Orthod., 8: 29-33, 1959.

Trauner, R. \& Obwegeser, H. The surgical correction of mandibular prognathism and retrognathia with consideration of genioplasty. II. Operating methods for microgenia and distoclusion. Oral Surg. Oral Med. Oral Pathol., 10(9):899-909, 1957.

Turvey, T. \& Schardt-Sacco, D. LeFort I Osteotomy. In Fonseca, R.; Betts, N. \& Turvey, T. Oral and Maxillofacial Surgery. Vol 2. Philadelphia, WB Saunders Company, 2000. pp.232-48.

Van Sickels, J. E. \& D'Addario, M. Esthetic Options with Two Jaw Surgery. Oral Maxillofac. Clin. N. Am., 19:423-33, 2007.

Wolford, L. M.; Chemello, P. D. \& Hilliard, F. W. Occlusal plane alteration in orthognathic surgery. 
OLATE, S. \& CHAVES NETTO, H. D. M. Manipulación del plano oclusal en cirugía ortognática: consideraciones faciales. Int. J. Odontostomat., 4(1):23-32, 2010.

J. Oral. Maxillofac. Surg., 51(7):730-40, 1993.

Wolford, L. M.; Chemello, P. D. \& Hilliard, F. W. Occlusal plane alteration in orthognathic surgery--Part I: Effects on function and esthetics. Am. J. Orthod. Dentofac. Orthop., 106:304-16, 1994.

Wolford, L. M. \& Hilliard, F. W. The surgical-orthodontic correction of vertical dentofacial deformities. J. Oral Surg., 39(11):883-97, 1981.
Dirección para correspondencia:

Sergio Olate

Depto. de odontología Integral

Facultad de Medicina

Universidad de La Frontera

CHILE

Email: solate@ufro.cl

Recibido : 11-11-2009

Aceptado: 09-02-2010 